\title{
La mujer en tradiciones orales africanas: ¿Transmisora o figura subversiva?
}

\author{
PAULINA MARÍA BARRIOS GIORDANO \\ El Colegio de México
}

\section{Resumen}

Este artículo discutirá la función filosófica y social de tradiciones orales africanas desde una perspectiva de género. En primera instancia se llevará a cabo una discusión acerca de la importancia filosófica y social de la tradición oral en África. Se argumentará que el concepto de literatura en África incluye a la oralidad y que además juega un papel importante en la transmisión y el cuestionamiento de conceptos filosóficos y sociales. Finalmente se analizará el papel que juegan las mujeres en las tradiciones orales africanas, enfocando la discusión en el caso particular de la mitología y el folclor de los swahilis.

Palabras clave: literatura, mitología, África, género, filosofía

\begin{abstract}
This article will discuss the philosophical and social function of African oral traditions from a gender perspective. The first section will include a discussion on the philosophical and social importance of oral traditions in Africa. It will be argued that orality should be included in the definition of African literature, and it plays an important part in the transmission and questioning of philosophical and social concepts. Finally, women's role in African oral traditions will be analyzed, focusing on the particular case of Swahili mythology and folklore.
\end{abstract}

Keywords: literature, mythology, Africa, gender, philosophy 
Art is not a mirror held up to reality but a hammer with which to shape it.

- Bertolt Brecht

a narración juega un papel central en la vida humana ya que encarna nuestra interacción social y nuestro conocimiento propio. Como indica Barbara Hardy:

We dream in narrative, day-dream in narrative, remember, anticipate, hope, despair, relieve, doubt, plan, revise, criticize, construct, gossip, learn, hate, and love by narrative. In order really to live, we make up stories about ourselves and others, about the personal as well as the social past and future (cit. en Finnegan 1998: 1).

La narración está presente en todo lo que nos rodea, cuando leemos un libro, escribimos nuestros pensamientos en un diario, le escribimos una carta a un ser querido, o cuando nuestros padres y abuelos nos cuentan una historia de su niñez Por lo tanto, la narración se compone de lo oral y lo escrito, que se complementan y forma parte de la expresión no sólo diaria, sino también artística y literaria. Al hablar de narración literaria desde la perspectiva del Occidente, muchas veces se olvida que incluye la narración oral y ésta se designa como externa a la literatura. Sin embargo, esta es una conclusión errónea, sobre todo en contextos africanos donde la tradición oral es parte de la producción literaria.

La tradición oral juega un papel importante en las poblaciones de África y dicha importancia se ha mantenido de manera consistente, sobre todo en la mitología y el folclor del continente. El papel de estas expresiones literarias, entre ellas los cuentos folclóricos, los mitos y las épicas, va más allá del entretenimiento. Por medio de ellas los narradores, y especialmente las narradoras, educan a los niños y expresan la filosofía detrás de los cuentos y las prácticas culturales de su sociedad. Estos cuentos tienen una doble funcionalidad. La primera es transmitir las enseñanzas y la filosofía de la cultura que los crea, pero también pueden tener un segundo papel de subversión de estas mismas prácticas u ofrecer un medio para escapar de la estructura social. Especialmente en 
el caso de las mujeres, quienes pueden crear un medio, muchas veces mágico, en el cual son libres para transformar su destino. La meta de este artículo será llevar a cabo una discusión acerca de la oralidad en varias culturas africanas, así como la función particular de las mujeres en ella. ¿Debe de incluirse la oralidad dentro de la definición de literatura o no? ¿Cuáles son las funciones filosóficas y educacionales de esta oralidad? ¿Qué papel juegan las mujeres en la narración de tradiciones orales y dentro de las narraciones mismas? Se buscará responder a estas preguntas, así como aterrizarlas en el caso concreto de cuentos folclóricos y mitos de los swahili, grupo poblacional de la costa este de África.

\section{La tradición oral en África}

África se ha designado como 'el continente oral', muchas veces a expensas de su expresión escrita, por la importancia de lo oral en las tradiciones africanas. Finnegan (2007: 1-12) desarrolla este aspecto al hacer una revisión desde los años sesenta y de los estudios etnológicos llevados a cabo en África, en los que se demostraba la importancia de la tradición oral en la transmisión de filosofía, prácticas culturales, religión, etc., en las culturas africanas (e. g. Griaule (1965) y su entrevista con el sabio Ogotemmeli), hasta estudios recientes acerca de la oralidad en África y lo que ésta representa en el continente. Finnegan hace una recopilación detallada de los autores más recientes que tratan con la oralidad en África por lo que es conveniente citar la sección completa:

Commentators from all sides concur on the significance of the oral and the spoken word. Africa has been termed "the oral continent par excellence" with "orality" as the means by which "Africa made its existence, its history, long before the colonial and imperial presence of the west manifested itself" (Gunner 2004:1). Christine Seydou draws attention to "the prevailing importance of the spoken word in [African] cultures" (2004:117) and Philip Peek and Kwesi Yankah reiterate a widely accepted position when they emphasize "the primacy of the human voice and of the exchange of life through words" (Peek and Yankah 2004:xii) (Finnegan, 2007: 1). 
Por lo tanto, la presencia de la oralidad es claramente un aspecto fundamental de varias culturas africanas. Parte de esta oralidad está conformada por los mitos, las épicas y los cuentos folclóricos que ilustran tanto el pasado cuanto el presente de las comunidades que las producen y que les dan vida. ${ }^{1}$ La representación de cuentos y mitos encapsula lo que Peek y Yankah describen acerca de la primacía de la voz humana y el "intercambio de la vida a través de las palabras". Por medio de ellos los narradores y las narradoras logran dar vida a sus creencias, sus vivencias diarias y logran también un cuestionamiento de las éstas.

Aun así, se tiene que resaltar que esto no debe de restarle importancia a la literatura escrita ni tampoco usarse como una minimización de la literatura africana escrita. Como explica Finnegan (2007: 2), lo escrito también juega un papel importante en tradiciones africanas, eventualmente las diferencias entre lo escrito y lo oral se disuelven. Este punto lo enfatiza Julien (1971: 22), quien argumenta que las culturas no son ni enteramente ni exclusivamente orales ni escritas. Ambas formas de expresión se complementan; hay una coexistencia entre ambos que caracteriza a la mayoría (sino es que todas) las culturas humanas. Es decir, enfocar esta discusión en la oralidad en África no quiere decir que no haya una presencia escrita ni que falte un intercambio entre ambos aspectos de la literatura; al contrario, como se discutirá más adelante, la literatura implica tanto lo oral como lo escrito. Por ello, el concepto de tradición oral implica diversos elementos y se ha discutido en varios trabajos (Wiredu [2009], Viveros y Djogbénou [2009], Finnegan (2007), Lorentzon [2007], Wa Thiong’o [2007],

1 Dentro de los estudios del folclor y la mitología hay debates que rodean las definiciones de mito y cuento folclórico. De manera muy general el mito se asocia con elementos históricos y mantiene elementos mágicos o surreales que se tratan como elementos naturales, se centra por lo general en una temática divina o en relación con fenómenos naturales. La épica también se basa en elementos históricos, incluyendo elementos mágicos, pero centrada en un héroe y las hazañas que debe completar. El cuento folclórico, por su parte, se diferencia por no necesariamente estar enfocado en un evento histórico, también contiene elementos denominados como fantásticos, sus temas son más relacionados a vivencias diarias y menos a elementos divinos o a explicaciones de fenómenos naturales. Varios trabajos se han enfocado en discutir acerca de definiciones de mitología y folclor y su relación con la literatura (Dorson, Lévi-Strauss, Maranda, Propp, entre otros). 
entre otros). Por medio de una revisión de algunos de sus trabajos se llegará a la descripción de lo que en este artículo se denominará oralidad y tradición oral.

Kwasi Wiredu define a la tradición oral como una transmisión de ideas de una generación a otra, por medio de la palabra oral y cualquier otro medio que no sea escrito (Wiredu 2009: 10). Presenta a la oralidad africana como filosófica, enfatiza su función como medio de transmisión y argumenta que está presente en varias instancias de la vida de poblaciones africanas. De manera similar, Amadou Hampaté Bâ argumenta que las tradiciones orales africanas no se limitan a la mitología y al folclore, sino que:

La tradición oral es la gran escuela de la vida, que recubre y abarca todos los aspectos. Ella puede parecer un caos a aquel que no penetra su secreto y desconcertar al espíritu cartesiano habituado a dividir todo en categorías bien definidas. En ella, efectivamente, lo espiritual y lo material no están disociados. Al pasar de lo esotérico a lo exotérico, la tradición oral sabe ponerse al alcance de los hombres, hablarles según su entendimiento y desarrollarse en función de las actitudes de ellos. Es todo a la vez - religión, conocimiento, ciencia de la naturaleza, iniciación de oficios, historia, diversión y recreación-, con toda clase de detalles que pueden permitir siempre subir hasta la unidad primordial. Fundada sobre la iniciación y la experiencia, la tradición oral abarca al hombre en su totalidad, y por eso se puede decir que contribuye a crear un tipo de hombre particular y a esculpir el alma africana (Viveros y Djogbénou, 2009: 11-12).

Así, por medio de los autores citados se puede determinar que la oralidad en la mayoría de las poblaciones africanas representa un medio importante de transmisión artística, educativa y filosófica.

Otro aspecto importante en los estudios de la oralidad es el concepto que se utiliza, justamente buscando alguno que ayude a englobar todo lo antes descrito. Uno de los términos propuestos es el de oratura, sugerido por el lingüista Pio Zirimu, en los años setenta, en un intento por responder a esta concepción errónea de las expresiones orales como menores a las escritas, con lo queo buscaba también contestar el término de literatura oral (Wa Thiongo, 2007: 4). El nuevo concepto no fue muy profundizado debido a su asesinato en los años 
setenta. Ngugi Wa Thiong’o lo describe como el uso de la aseveración como el medio estético de expresarse (2007: 4). Otro autor que ha trabajado el término es Pitika Ntuli, quien lo describe como:

Orature is more than the fusion of all art forms. It is the conception and reality of a total view of life. It is the capsule of feeling, thinking, imagination, taste and hearing. It is the flow of a creative spirit" (1988: 215). He expressed the interconnectedness of phenomena in terms of a "beginning come full circle on a higher plane (215) (cit. en Wa Thiong'o, 2007: 5).

Así, por medio de esta terminología los autores buscan obtener una concepción más inclusiva de la tradición oral en contextos africanos. Esta discusión terminológica muestra otro aspecto importante de la oralidad: su concepción como literatura.

\section{Lo oral como literatura}

Esta discusión es pertinente al actual artículo ya que se busca argumentar que las expresiones orales que se analizarán al final del artículo son expresiones literarias y por ello pueden ser analizados como tal. Se considera que llevar a cabo esta discusión filosófica y literaria es importante para integrar los mitos, cuentos y épicas descritas a lo largo del artículo en la concepción de literatura, para así llegar a una expresión más inclusiva e importante como un diálogo entre las expresiones escritas y orales. Julien enfatiza este punto al argumentar que es importante no aislar la oralidad, verlo como algo singular o separada de la escritura, sino más bien que las expresiones habladas y escritas son modos de lenguaje y que ambos son nuestros cuando tenemos los medios para producirlos (1971: 24). Afirma también que al ver su interacción en géneros literarios, no debería de ser con el esfuerzo de probar o refutar la autenticidad cultural, sino de apreciar la literatura como un acto social y estético (1971:24).

Por lo tanto, el concepto de la oralidad no se considera como algo aparte de la literatura, sino que se incluye en ella; hay un diálogo constante entre lo escrito y lo oral. Esto se ve reflejado, por ejemplo, en el concepto de la novela afri- 
cana, que ya se venía expresando en las tradiciones africanas al encontrarse una tradición fuerte de épica oral y también de tradiciones escritas (Sullivan, 2006: 180). Sullivan indica que aunque sí se trajo un concepto occidental de la novela, se ha ido adaptando y ha ido incluyendo diferentes aspectos de la tradición literaria africana. Una parte importante es la influencia de la tradición oral en las novelas escritas africanas. Sullivan cita a Ibrahim Yaro Yahaya para explicar que dentro de los cuentos folclóricos se incluyen canciones para mantener la atención de la audiencia, aumentar la estructura dramática de la historia y conllevar mensajes que de otra manera no están disponibles en el texto, y argumenta que lo mismo se puede observar en la novela africana (cit. en Sullivan, 2006: 180). Esta influencia se lleva hasta casos donde las novelas mismas se estructuran como cuentos folclóricos o mitos. Por esto Sullivan argumenta que las novelas muchas veces no son producto de una persona individual, sino de él o ella y su comunidad (2006: 181). Es así que en la novela, una base importante de la literatura, se refleja este intercambio constante entre oralidad y escritura en África, así como la centralidad de lo oral en la literatura africana.

La oralidad tiene una importancia en la expresión literaria escrita y también tiene un peso por sí misma. Es decir, los cuentos, las poesías, épicas y demás expresiones orales también son considerados como literatura en sí mismos. Lorentzon (2007) lleva a cabo una discusión detallada acerca de la oralidad y la literatura, por medio de ella demuestra las diferentes tendencias dentro de los estudios de oralidad en África. Uno de los aspectos importantes de la oralidad es su representación. Finnegan enfatiza que la tradición oral depende de su narrador o narradora, quien formula esta expresión oral para una ocasión específica (2007: 78). Es decir, por medio de esta representación, el cuento cobra una vida propia, aunque sea un producto de una sociedad y de un narrador o narradora. Esta representación es uno de los elementos que nos permite designarlo como literatura, ya que esto implica que tiene un autor o una autora.

Pero Lorentzon argumenta que la representatividad de los cuentos en sí no es suficiente para explicar que la oralidad es literatura, ya que según esta concepción cualquier "texto" que se represente sería literatura (incluyendo discursos políticos, por ejemplo). Para responder a esta problemática el autor sugiere que varios elementos deben de estar presentes para que una expresión oral sea 
literaria. La primera es que sea "citable"; es decir, que tenga un elemento que transcienda a través del tiempo, lo que se cita en la representación, eso es su aspecto literario: "it is the story that is 'quoted' in each performance" (Lorentzon 2007: 8). Un segundo elemento es finalization: Seitel describe este término como "an artistic achievement by individual performers [and] the achievement of engaged, informed audiences [...] finalization is the tool for creating and interpreting artistic visions of communal cultural potentials, for achieving individual and collective insight" (cit. en Lorentzon, 2007: 9). Por lo tanto, es por medio de una presencia de todos estos aspectos que uno puede determinar que una tradición oral es literaria. Como se puede observar en las citas anteriores, parte de lo que hace que la tradición oral sea una expresión literaria es el mensaje que conlleva, sobre todo la función filosófica, artística y social que tiene.

\section{Función filosófica y social de la oralidad}

Como ya se ha aludido anteriormente, la literatura cuenta con una funcionalidad que va más allá del entretenimiento de su audiencia. En general, los cuentos folclóricos son contados a los niños y jóvenes de una comunidad y por ello conllevan un importante peso filosófico y educativo. Wiredu argumenta que la tradición oral es una fuente de sabiduría africana; es por medio de los diferentes cuentos folclóricos, mitos, poesías y épicas, entre otros, que se expresa la filosofía de la población en cuestión. Wiredu comienza su discusión con los proverbios y argumenta que frecuentemente tienen un significado filosófico, al contener no sólo consejos prácticos, sino también conceptos filosóficos y sociales (2009: 10). En el caso de algunos proverbios africanos acerca de la mujer, por ejemplo, se pueden ver las diferentes maneras en que se concibe a la mujer y su función en la sociedad. En el caso de un proverbio de Kenia, por ejemplo, expresa que "the man may be the head of the home, the wife is the heart", con lo cual se determina que la mujer representa el aspecto emocional del hogar, mientras que el hombre es el sustento del hogar. Otro dice: "Married women are like elephant tusks: you don't touch them". Este proverbio conlleva un peso ético al determinar que los hombres deben de respetar las esposas de otros al 
no acercarse a ellas, implica que hay una cierta estructura social, la familia, que debe de ser respetada.

Además de los proverbios, Wiredu menciona el resto de la oralidad, los acertijos, la poesía, los cuentos folclóricos, los mitos, las épicas, los cantos funerarios, etc. Argumenta que, contrario a la percepción que se tiene de que la filosofía se expresa únicamente por escrito, en realidad todas las tradiciones de conocimiento y filosofía tienen un cierto elemento de oralidad. Determina que el mismo lenguaje tiene una influencia sobre el pensamiento filosófico de la población que lo utiliza; la sintaxis y el vocabulario predisponen a pensar en cierta manera.

La oralidad entra en el momento en que una idea es tratada como un objeto (Wiredu 2009: 12). Esto puede ejemplificarse en los cuentos folclóricos y los mitos. En ellos se expresan concepciones filosóficas de las poblaciones que los producen, la manera en cómo se percibe al individuo, a la sociedad, el concepto de masculinidad y feminidad, la importancia o no de la familia, etc. Por medio de ellos se llega a una percepción acerca de la comunidad en cuestión. De manera similar Viveros y Djogbénou argumentan que las manifestaciones orales y escritas no se deben de excluir (2009: 11). En realidad ambos coexisten y en algunas ocasiones se llegan a complementar. En el caso de los cuentos folclóricos, las épicas y los mitos, claramente son de origen oral pero éstos ya se van transformando en una tradición también escrita al irse transcribiendo para su transmisión hacia otras culturas o para introducirlos en el contexto urbano o la educación "formal".

Esto se puede ver reflejado en el cuento folclórico swahili que se titula The Trapper, que se enfoca en un cazador que atrapa muchos animales, pero se los roba un monstruo. Un día decide atacar al monstruo pero éste se da cuenta y lo persigue. El cazador corre hacia su casa y le grita a su esposa que lo ayude a defenderse, ella toma un pan que tenía en la cocina y lo avienta al monstruo, que lo come y muere instantáneamente. El esposo le pregunta sospechoso a su esposa si había envenenado el pan, pero ella le explica que no, que era un pedazo de pan normal pero que lo aventó al monstruo pensando que era mejor que se comiera el pan que a su esposo. En ese momento el maestro para y les explica: "Evil spirits die in the face of virtue and courage" (Knappert, 1970: 140). Aquí 
se puede ver varios elementos de lo que se describió anteriormente. En primer lugar el cuento refleja las prácticas sociales de la población, el hombre es el que va de caza y la mujer es la que se queda en el hogar cocinando. En segunda instancia, se ven reflejadas las relaciones de género entre el esposo y la esposa, y en tercer lugar se muestra el mensaje moral del final del cuento, la virtud y el valor de la mujer salvan a su esposo. Así, aunque el hombre sea el que trabaja y mantiene el hogar, la mujer es la que lo defiende y es por medio del trabajo honesto de ambos que logran enfrentarse a enemistades o cualquier aspecto negativo que puede ser representado por el monstruo.

Por lo tanto, la tradición oral conforma una parte importante de la base filosófica de la comunidad y de su transformación. También cumplen un papel fundamental como guías y como medios de aprendizaje. Carol Boyce Davies argumenta que la tradición oral es fundamental para la definición de la estética y las dinámicas psicosociales de un grupo (cit. en Sougou, 2008: 28). Es por ello que los cuentos folclóricos son especialmente importantes para la educación de los niños y jóvenes que los escuchan. Estos cuentos conllevan un peso filosófico y una función social, al educar a su audiencia sobre las prácticas sociales y los papeles que juegan cada uno de los individuos en la sociedad.

Ngwainmbi, hablando acerca del poder de la tradición oral, llega a argumentar que estos cuentos enseñan tolerancia y respeto, lo cual lleva a que los niños expuestos a estas tradiciones muestren mayor tolerancia a otras comunidades y creencias. (2007:20). Aunque es importante evitar este tipo de generalizaciones, ya que no necesariamente una mayor exposición a los cuentos y a la mitología equivale a una mayor tolerancia, su comentario es pertinente porque apunta hacia la centralidad de la tradición oral en la educación de los niños y jóvenes de varias comunidades africanas. También es relevante ya que resalta un aspecto importante de varias culturas africanas: las ideas de comunidad y reciprocidad que se ven reflejados en los cuentos folclóricos. Todos los individuos en la sociedad tienen un papel que cumplir y, al cumplirlo, se benefician a su vez del trabajo de otros; es un trabajo en conjunto en el que todos se benefician. Cuando este balance se rompe vienen los momentos de crisis en los cuentos y el final feliz no llega sino hasta que se recupera este balance. Este sentido de 
reciprocidad es por lo que Ngwainmbi argumenta que estos cuentos llevan a una mayor tolerancia y respeto.

En este caso Ngwainmbi se refiere a la comunidad Kom de Camerún. Wiredu (2009) menciona esta misma importancia de los cuentos folclóricos entre los Akán de Ghana; Abbas H. El-Nour (2011), en relación al norte de Sudán, y Sougou (2008), en relación con cuentos folclóricos de Senegal. Por lo tanto, este concepto del cuento folclórico como medio educativo está presente en varias culturas africanas. Abbas H. El-Nour lo resume claramente de la siguiente manera: "That room was our first classroom. There we learn about right and wrong, especially dreams and disappointments, reality and myth: that lying will take you straight to hell fire, or even worse, the su'luwwa might eat you alive [...] when you do good, you are sure to live happily ever after" (2011: 171). Ella argumenta que en las áreas rurales estos cuentos conforman la base educativa de los niños y jóvenes:

In the narration of huja to children, a moral twist and a didactic conclusion are always expected. The children learn the intended lesson by identifying with the hero of the tale and they equate his behaviour with what they should do in order to be admired and rewarded by the society (Hurreiz 1977: 33). The older members of the community use these tales as "social stories" to indirectly and craftily inject the desired moral values according to the conventions of the community or the tribe, and to warn (the listeners) against ignoring or breaking them (Abbas H. El-Nour, 2011:174).

Por medio de la representación del cuento se logran exponer diferentes puntos acerca de la interacción social de la población en cuestión, así como cuestionamientos filosóficos y/o educativos. Sus enseñanzas se logran por medio de la representación de los cuentos y de su discusión.

Es aquí donde se introduce uno de los papeles que juegan las mujeres en relación con la tradición oral en contextos africanos. En la mayoría de las tradiciones africanas (Musiyiwa, 2009) las mujeres narran los cuentos folclóricos. Aunque hay grandes narradores que son hombres, sobre todo en relación con las épicas africanas, los cantos y los rituales, la mayoría de ellos fueron introducidos a la narración y a los cuentos folclóricos por sus abuelas. Como indica 
Abbas H. El-Nour, son principalmente las ancianas de las comunidades las que relatan estos cuentos. Musiyiwa explica que la oralidad tiene como objetivo central socializar a los individuos en la vida de una comunidad (2009:7).

Este aspecto filosófico de la tradición oral es algo que se ha llegado a criticar, sobre todo cuestionando la inmovilidad que esto implica en la misma filosofía que se transmite; asimismo ha habido una crítica a la falta de individualidad de este concepto de "filosofía africana", apuntando el peligro de llevar a cabo generalizaciones. Hountondji es uno de los críticos más fuertes del concepto de etnofilosofía, quien afirma que la filosofía africana no es un pensamiento colectivo e implícito, lo cual cuestiona profundamente lo que determina como unanimismo, es decir, sobrevaluar la unanimidad de las poblaciones africanas y argumentar que hay una única filosofía africana (2004: 530). Argumenta en contra de la concepción etnológica de la filosofía africana, en cuanto a que es reducida a una visión del mundo colectiva e irreflexiva (1977: 134). Finalmente explica que para que puedan haber discusiones filosóficas africanas, tiene que incluirse un aspecto escrito, sobre todo para la transmisión de ideas, utilizando como ejemplo el caso de los filósofos griegos, quienes fueron transcritos por sus alumnos u otros (1977: 135). En cuanto al aspecto de la filosofía escrita, lo desarrollado anteriormente demuestra que no es necesario que la filosofía esté escrita para ser transmitida, y que aún la filosofía escrita, como argumenta Wiredu, tiene una base importante en la oralidad.

La crítica en contra de suponer una única filosofía africana, generalizada y estática, se considera una crítica central y forma parte de la discusión de este artículo. Afirmar que una filosofía se puede transmitir de una generación a otra por medio de una tradición oral, puede implicar que dicha filosofía no cambia, que sigue siendo pertinente para la sociedad, de la misma manera que al iniciar la tradición oral. $\mathrm{Al}$ argüir que estas tradiciones orales se refieren a la filosofía de una población, en general también puede dejar poco lugar para la expresión individual. Por ello aquí se argumenta que estos conceptos filosóficos y sociales expresados en las tradiciones orales no son estáticos ni son generalizables a todas las poblaciones africanas, sino que son expresiones reflexivas y particulares a las sociedades que las expresan, dejando también lugar para el narrador o la narradora como individuo. 
La transmisión de la oralidad de generación en generación no implica que sea estática: los símbolos que las conforman, la retórica y el ethos invocado hace a cada una de estas expresiones particular (Mafeye 1997: 16). Pero aun estas expresiones cambian según el contexto social e histórico (1997:16). Igualmente, los elementos filosóficos y ontológicos expresados no son elementos que se creen de manera uniforme a través de la sociedad en cuestión. Wiredu ejemplifica esto utilizando tradiciones orales de la comunidad Akán y concluye que "there was no uniformity of thought in the traditional society under discussion [and] a pattern of thought in an oral tradition need not be communal; it may be the outcome of an original and nonconforming way of thinking" (2009: 11). Por lo tanto, el considerar a la tradición oral como un elemento filosófico y educativo no implica que ésta sea estática ni que sea algo que se transmita de persona en persona y de generación y generación, sin ningún tipo de reflexión y cuestionamiento. Esto se ve reflejado también al llevar a una crítica de los proverbios mencionados anteriormente. Según lo indicado en el primer proverbio, las mujeres no tienen la capacidad intelectual de dirigir su hogar, ellas deben entonces limitarse a su papel de madres y esposas, y dejar el manejo del hogar para el esposo. Esto no refleja lo visto en el cuento swahili que comentamos, donde la mujer muestra astucia y valor al defender a su esposo del monstruo. Dentro de la misma tradición oral se pueden encontrar debates y contradicciones que muestran que no es una visión única, estática, ni unilineal.

Por medio de la voz femenina como narradora y como personaje dentro de los cuentos folclóricos, se muestra lo dinámicos y contestados que pueden ser sus elementos filosóficos y educativos. Esto se hace por medio de la apropiación de las narraciones que con ello proporcionan un área para que los individuos lleven a cabo cuestionamientos sociales y filosóficos particulares, diferentes a lo ya establecido en el cuento. De la misma manera, los conceptos filosóficos y sociales transmitidos no son estáticos: cada vez que se cuentan se hace en un contexto y audiencia específicos, por ello cada expresión es particular y pueden verse justificaciones o subversiones en un contexto en concreto. Por ello, la filosofía y las enseñanzas impartidas entran en un diálogo con las narradoras y con su audiencia. En particular a las mujeres les ofrece un espacio propio, un medio 
en el cual construir y adaptar sus oportunidades de crecimiento y renovación (Sougou, 2008: 26).

\section{Las mujeres como narradoras y compositoras}

Como ya se discutió, un aspecto fundamental de los cuentos folclóricos es el narrador. Todo el cuento y su efecto dependen de esta persona, de su capacidad de expresar las emociones descritas y de poder describir los eventos que se van presentando. Por ello, su designación es extremadamente importante, y hay diferentes maneras de definir a la persona indicada. Hay especificidades para cada cultura en cuanto a las convenciones de lo que implica ser narrador o narradora de tradiciones orales. Finnegan explica que en algunas sociedades esto parece ser muy flexible, y en otras es más definido. En algunas poblaciones las mujeres son consideradas las más dotadas, sobre todo las de mayor edad (Abbas H. ElNour, 2011: 174). En el caso de varias tradiciones africanas, entre ellas comunidades del Norte de Sudán y de Senegal, los grupos étnicos shona, zulu, ndebele, xhosa y gikuyu, las mujeres mayores generalmente se encargan de narrar los cuentos folclóricos, y los hombres, los cuentos más históricos.

Sin embargo, es importante no generalizar esta distinción ni tomarla como muy estricta ya que en algunas tradiciones las mujeres también se encargaban de transmitir otros aspectos de la tradición oral. Musiyiwa (2009) y Abbas H. El-Nour (2011) enfatizan la importancia de las abuelas no sólo en la acción de narrar los cuentos y mitos, sino también en su selección. La centralidad de las mujeres en la tradición oral se ve también reflejada en la cultura swahili. Joseph Mbele argumenta que las mujeres swahilis juegan un papel importante tanto en la producción poética como en su preservación. Asimismo muestra que la presencia femenina en la tradición oral es inclusiva y que sobre todo se enfoca no sólo en preservar la tradición literaria de su cultura, sino también en producirla: "A great part of the Swahili culture is in the hands of women and particularly elderly, even very old women [...] many Swahili poets have been ladies of high rank and this may have something to do with the very high position of women in Kiswahili verse [...] women have also been prominent in the work of preserving the literature" (Allen cit. en Mbele, 1996: 74). A lo largo de su artículo 
Mbele argumenta que la presencia femenina en el poema que analiza, Wimbo wa Miti ("Canción sobre un árbol”), va más allá de la narradora ya que también es crítica. Por medio del poema se lleva a cabo una crítica social, como, por ejemplo, la violencia, que se consideran como parte de un idealismo heroico. Por lo tanto estas mujeres no fungen solamente como productoras y preservadoras de tradiciones orales, sino también como sus críticas.

La función de las mujeres como narradoras refleja también la estructura de las sociedades en las que ellas se desarrollan. Tomando en cuenta que en la mayoría de estos casos, la mujer funge como narradora en el contexto del hogar demuestra también la división de las tareas entre hombres y mujeres. En los casos descritos aquí, poblaciones que se encuentran en el oeste, norte y este del continente africano, las mujeres están sobre todo dentro del contexto del hogar. En este caso es importante tomar en cuenta que el elemento de entretenimiento puede también ser un elemento funcional en sí mismo. Es decir, las mujeres son las encargadas no sólo de educar a los niños, sino de cuidarlos y llevar a cabo tareas del hogar, y esto muchas veces lo logran por medio de narrar cuentos. Como describe la autora y profesora Juliana Makuchi:

It was a good diversion that my mother always used because [. . .] when you have ten hungry children waiting to eat and some are crying and some sleeping and some full of boundless energy, using folktales, I think, served many purposes. It was a very gendered weapon that my mother used, I would say, because we would enjoy the stories, and at the same time it would divert our attention from hunger and from fatigue. Sometimes, when we were telling stories and my mother wanted us to shell groundnuts... or beans or stuff that would be used for a meal the next day or on the farm, she would pull a bag out and dump it in our midst and we'd work as we told stories (cit. en West, 2007: 55).

De aquí es importante rescatar dos aspectos: la primera es el contexto social de la narración y el segundo es el aspecto multilateral de las historias, es decir: la audiencia escucha y también participa. En cuanto al primer aspecto, se resalta de nuevo la función de la mujer como la narradora; demuestra un contexto hogareño, y una presencia de mujeres y niños. Esto apunta hacia el papel central 
que juega la mujer en la familia y en el trabajo del hogar. De la misma manera, se demuestra el aspecto social de estos cuentos: se utilizan para entretener a los niños y para incitarlos a participar, a que sean también productores de estos cuentos y no sólo que escuchen. Esto lleva a que poco a poco los niños se vuelvan a su vez transmisores de los cuentos con su propio matiz. Como dijo Mbiti en los años sesenta, cada persona que cuenta un cuento no sólo lo repite, sino que también lo crea, cada persona le agrega su propia originalidad y "frescura" (cit. en Scheub, 1999: 311). Es decir, estos cuentos no son estáticos; en estos contextos del hogar, el intercambio es entre las abuelas o las madres y los niños. Aun teniendo esto en cuenta es importante resaltar que aparte de este contexto hogareño de la narradora, también había y sigue habiendo una presencia pública de las mujeres como narradoras:

The importance of women in storytelling was recognized by the community, which sometimes composed special praises and praise names for them: They were, for instance, identified by titles such as sarungano (owner of the story) among the Shona; and umethi (narrator) among the Zulu, Xhosa, and Ndebele. Among the Luo, the pim (grandmother) was praised as one who was "cleverer than proverbs", because of her intimate knowledge of storytelling (Onyango-Ogutu and Roscoe 26). The content of women's stories was so diverse that it covered the entire spectrum of their societies' cultural life, including stories about the need to protect children's and women's rights, gender harmony, and family matters (Musiyiwa, 2009: 8).

De la misma manera Mbele menciona la presencia pública de mujeres como Zaharia, una poeta de sesenta años, que ha educado a intelectuales que trabajan sobre cultura y poesía swahili (Mbele, 1996: 75). Por lo tanto, las mujeres no sólo tienen una presencia como narradoras dentro del hogar, sino que también pueden llegar a jugar papeles centrales como narradoras públicas y como custodias de acervos literarios africanos.

Un medio público y privado donde la mujer juega un papel central es en las representaciones orales que acompañan ritos o momentos claves de la vida, es decir nacimientos, bodas, ritos de paso, funerales, etc. En estos ritos las mujeres juegan papeles cruciales como narradoras y compositoras, como en el caso 
de las bodas donde las mujeres no solamente participan en los cantos y los bailes de los ritos, sino que también los pueden llegar a crear. Los dos ejemplos que se desarrollarán aquí son el de la cultura wolof, grupo étnico situado al oeste del continente africano sobre todo en Senegal, Gambia y Mauritania; y el de los swahili, cultura originaria del litoral costero y las islas cercanas a la costa del este del continente, desde el sur de Somalia hasta el norte de Mozambique y las islas Comoro.

En el caso de las bodas wolof, Gueye (2010) se enfoca en el caso particular de su tía paterna, Seynabou Dieng o Yaay, quien canta y compone una expresión particular a las bodas wolof: el woyyi céet. Gueye argumenta que aunque las canciones parecen reforzar la subordinación de las mujeres, su tono sarcástico las hace políticas y las convierte en una protesta. Mientras las canciones informan sobre la socialización entre hombres y mujeres, por medio de ellas las mujeres también pueden criticar esta cultura que las marginaliza. Aunque la autora explica que estas canciones en particular se utilizan menos en las bodas desde la época colonial; en ellas se expresan las relaciones entre la novia y su nuevo esposo; entre la novia y las mujeres mayores de su familia, sobre todo su mamá, llevan a cabo una explicación y una crítica de las relaciones maritales; se cuestiona la práctica de la dote, etc. En general muestra un espacio literario donde la mujer puede protestar su situación, donde se le previene sobre ciertas prácticas que debe evitar y finalmente se le dan enseñanzas sociales. Es importante enfatizar que en este caso en particular la autora de la canción, Yaay, usa este espacio para recordarles a las mujeres acerca de sus derechos y enfatizar los deberes del esposo (Gueye, 2010).

De igual manera en los ritos de paso y las ceremonias matrimoniales de los swahili las mujeres participan en los cantos y encabezan los eventos de la boda relacionadas con la novia. En el caso de las niñas swahili hay dos ritos de paso principales: el unyago, entre los swahili de la isla de Mafia se lleva a cabo cuando las niñas menstrúan por primera vez (Caplan, 1976); y el vugo, entre los swahili de Mombasa, Kenia, donde se celebra la virginidad de la novia y donde juegan un papel central los cantos de las mujeres (Topan, 1995). Las canciones que enumera el autor aluden específicamente a una consumación exitosa del matrimonio. En ellas se refleja: el aspecto comunitario de una boda, que no es 
una alianza sólo entre individuos, sino entre familias y comunidades; la deuda que el novio tiene con la madre que se "paga" sólo parcialmente con la dote; se hacen referencias a la capacidad del novio para consumir el matrimonio; entre otros. Pero, al igual que en el caso del woyyi céet, en la actualidad la práctica de estas ceremonias ha disminuido, y se han dado paso a expresiones que se consideran más "modernas". Con estos dos ejemplos lo que se busca es mostrar otra figura que juega la mujer como narradora o compositora y su centralidad en varios ritos sociales.

Nnaemeka en su artículo acerca de oralidad y escritura femenina en África enfatiza esta presencia femenina no sólo como narradora y depositaria de tradiciones, sino también como compositora. Argumenta que es importante reconocer su centralidad como sujetos y como re-creadoras de tradiciones orales: al narrar estos cuentos las mujeres en ocasiones los transformaban para incorporar perspectivas más centradas en la mujer (Nnaemeka, 1994: 138). La autora enlaza la presencia femenina en la tradición oral con una fortaleza social y política de figuras de resistencia como Aoua Kéita. Kéita, mujer bamana que dirigió un movimiento de resistencia para la independencia de la África francesa ecuatorial, afirma que su fortaleza moral y su militancia vienen de las lecciones que aprendió de la presencia femenina en la tradición oral (Nnaemeka, 1994: 139). Aquí es importante no sólo enfatizar la centralidad de la mujer en la tradición oral, sino también que ella misma agrega su voz a la narrativa, también es compositora (o escritora). Como compositora la narradora tiene la capacidad de incluir críticas a las mismas historias que presenta, así como llamar a un cambio en las prácticas que ilustra y enseña.

Askew (1999) argumenta que las representaciones musicales ofrecen también un medio para llevar a un cambio social. La autora utiliza el ejemplo de los intérpretes de ngoma, quienes en el pasado y en el presente han logrado o evocado cambio social por medio de sus interpretaciones (que incluyen cantos, música y baile), las cuales llevan a la realineación del poder y el estatus en la sociedad swahili. Por lo tanto, ella argumenta que las representaciones orales constituyen una fuerza potente y potencialmente desestabilizadora y no se debe tomar como un simple entretenimiento (Askew, 1999: 77). Rosenberg aterriza este concepto en el caso de la música de Saida Karoli. Esta cantante forma parte 
de un círculo de representación que generalmente se designa a los hombres, es decir, la representación de épicas o sus equivalentes. El autor explica que aunque la cantante no recita épicas, sus canciones son representaciones equiparables (Rosenberg, 2012: 128). Como una intérprete femenina y líder de un grupo musical, Karoli actúa y canta en contra de un sistema de poder masculino en un medio de representación musical, jugando el papel de una intérprete tradicional pero también integrando su posición como cantante reconocida internacionalmente (2012: 128). Por lo tanto, esta licencia creativa que llegan a tener las mujeres en las representaciones orales las lleva a crear mensajes de prevención, de enseñanza, pero también de subversión.

Así, este papel de narradoras da a las mujeres la oportunidad de subvertir estas mismas tradiciones y de crear su propio mundo. En el caso de la mitología y el folclore, esto se hace por medio de la magia y la fantasía: se crea un mundo donde las mujeres pueden llegar a jugar un papel central, tomar decisiones propias y demostrar su valor no sólo por medio de sus tareas comunitarias, sino también por su belleza y su sabiduría. En el caso de las comunidades al norte de Sudán Abbas H. El-Nour explica cómo, en una comunidad patriarcal, el mundo de los cuentos folclóricos ofrece a las mujeres un espacio donde ellas son las dueñas de su destino y son capaces de crear sus propias historias. La autora argumenta que las narradoras utilizan los cuentos para vivir lo que se les niega en su vida diaria, un control sobre su destino, y logran retomar el control sobre sus vidas aunque sea por medio de un mundo imaginario y mágico (Abbas $\mathrm{H}$. El-Nour, 2011: 175).

El tener el control de transmitir y transformar la mitología y el folclor de una población les ayuda a las mujeres a sobrepasar la frustración de no tener control sobre su destino y las pone en un lugar central en la tradición y la cultura de una población. Como ya se discutió, esta oralidad incluye elementos filosóficos, sociales y educativos de los grupos poblacionales que las producen. Las narradoras, además de transmitir estas enseñanzas, unen los mundos de la fantasía y la realidad, y esto las lleva a ser una autoridad en la cultura de su sociedad (Abbas H. El-Nour, 2011: 176). Sougou, en su artículo sobre narrativas y mujeres en Senegal cita a Aissata Sidikou para explorar este punto: "the ritual space is a visual and aural environment where women demand to be seen 
and heard. It is a space that mirrors truth and untruth, reality and fantasy, thus challenging traditional knowledge and power that has constructed their existing feminine condition" (cit. en Sougou, 2008: 26). Por medio de este espacio las mujeres no sólo narran sus vidas diarias sino que las subvierten a través de los cuentos mitológicos que narran y las canciones que interpretan. Este papel de la mujer como narradora ha ido cambiando con el tiempo, lo que refleja cambios sociales e históricos que afectan las relaciones de género dentro de las diferentes culturas. Parte de ello es efecto de la islamización o la cristianización, la colonización y la modernización, lo que ha llevado a que ciertos ritos sociales ya no se lleven a cabo y a cambios paulatinos o drásticos en las relaciones de género (Gueye, 2010; Topan, 1995; Caplan, 1976; Askew, 1999; Musiyiwa, 2009; Mirza y Strobel, 1989).

\section{Las mujeres dentro de los cuentos}

Es importante tomar en cuenta que las relaciones de género cambian con el tiempo, reflejan la integración de nuevas religiones, nuevas poblaciones, eventos históricos, etc. Esto se refleja también en la mitología y los cuentos folclóricos de la población. Por ello, los papeles que juegan las mujeres dentro de los cuentos mitológicos y folclóricos africanos tienen dos funciones: la primera es para mostrar el ideal de la mujer o el contrario, la maldad de la mujer y el peligro que representa para la sociedad; y la segunda es la de criticar estas designaciones sociales.

Esto se ve reflejado en el caso de varias tradiciones mitológicas en África que se revisarán brevemente para llevar al caso concreto de los swahili. En el caso de los cuentos luo, Ayodo (1994: 123) argumenta que los personajes femeninos cumplen dos funciones: por una parte fortalece la estructura social bajo la cual ella se ve limitada a tareas domésticas y a una posición de marginalización; y en una segunda instancia muestran la responsabilidad social que la mujer tiene con su sociedad. Pero al mismo tiempo las mujeres logran vencer las limitaciones patriarcales al cumplir con sus responsabilidades y así poderlas atacar desde dentro del sistema. A través de los cuentos que revisa, Ayodo apunta hacia un mensaje constante a través de casi la totalidad de la oralidad: el de la 
fuerza interior de las mujeres y su uso del sentido común para responder a las problemáticas con las que se enfrentan ellas u otros personajes en los cuentos (Ayodo, 1994: 123). Por lo tanto, en este caso se puede observar que dentro de los cuentos las mujeres enfatizan la estructura social y las concepciones filosóficas y sociales que las mantienen en esa posición, pero también las cuestionan por medio de sus acciones o sus expresiones.

El siguiente ejemplo es el de los cuentos folclóricos etíopes. Rajendra Prasad (2007: 100) argumenta que a través de ellos se observa una justificación de la superioridad masculina. Utiliza cuatro cuentos cortos para demostrar que a través de ellos se ve reflejada una oposición binaria entre hombres y mujeres, donde las mujeres siempre se muestran como inferiores, sin integridad humana, con una sexualidad incontrolable, y sobre todo siempre enfatizando su falta de autocontrol (2007: 107). En este caso se observa el uso de cuentos folclóricos y mitos para transmitir enseñanzas acerca de los papeles de género y que justifican un sistema sociopolítico patriarcal. En la revisión de este artículo no se incluyen ningún ejemplo donde se subviertan dichas enseñanzas, ya que en todos los cuentos a las mujeres se les festeja por cumplir su papel o se les castiga por no hacerlo.

En última instancia se retomarán los cuentos recopilados por Sougou en Senegal. En ellos se ven reflejadas temáticas similares a las que se descifran entre los cuentos de la costa swahili, en parte por la influencia islámica sobre ambas regiones geográficas. En su artículo el autor hace un desglose de cinco cuentos y por medio de ellos identifica no sólo los mecanismos y las concepciones filosóficas que definen los papeles sociales de las mujeres, sino también su manera de subvertirlos. A través de los cuentos se muestran diferentes problemáticas específicas a las mujeres, como la poligamia, el problema de la educación para niñas, el matrimonio y la rivalidad femenina. La manera en que las jóvenes narran los cuentos y las decisiones que toman los personajes reflejan no sólo una crítica de la filosofía que está detrás de ciertas prácticas, sino también una subversión de éstas. Un ejemplo es el cuento titulado "Educación para niñas": allí se narra un accidente en el que unas niñas sufren heridas al caer en un pozo después de ir en busca de agua, por lo que el rey dictamina: "boys and girls alike will go to school or the daara... From now on, strong men will go to the far bore-holes or 
wells to draw water for sale in the village. Any woman sending her daughter to fetch water will be banished from the village" (Sougou, 2008: 30). Estos cuentos narrados en una época moderna por mujeres jóvenes muestran una exigencia por una equidad de género, por romper con estereotipos acerca de la mujer y así llevar a un cambio social en su percepción.

En el caso de los cuentos swahili, los personajes principales son generalmente hombres y la presencia femenina se ve generalmente en el papel de la esposa, la esclava, la reina o como la epítome de la tentación. A través de su mitología y folclor se pueden ver diferentes representaciones de la mujer donde casi siempre funge el papel de intermediaria entre el poder (ya sea un poder físico o un poder mágico) y su comunidad, o bien como poseedora de ese poder, y en este caso generalmente es representada como una figura de maldad. Para llevar a cabo un análisis más profundo se hará una revisión de tres cuentos y una épica, para así incluir dos formas literarias. Todos los textos se obtuvieron de antologías mitológicas y fueron originalmente representaciones orales recopiladas y transcritas ya sea por Edward Steere (siglo XIX) o Jan Knappert (siglo $\mathrm{XX}$ ). En el primer caso, se revisará el caso del cuento titulado "The Girl with One Hand", cuyo personaje central es una joven; el segundo es "Ali, the Sword and the Secret Charm", cuyo héroe es un hombre; "The Island of the Snake and the Land of Gold", cuento donde también juega el papel central una mujer, y finalmente "La Épica de Liongo".

"The Girl with One Hand" comienza con la muerte de los padres de la joven y su hermano, quienes al morir les preguntan a cada uno de sus hijos qué prefieren: si sus bendiciones o sus bienes. El hermano pide los bienes materiales, mientras que la hermana pide las bendiciones primero de su padre y después de su madre. Esta elección repercute en el resto de la historia marcando el papel que jugará cada uno de los hermanos y mostrando la filosofía detrás de cada uno de ellos. De la misma manera refleja las relaciones de género entre los swahili, sirviendo como la pauta para un comentario acerca del papel de la mujer a lo largo del cuento. La costa este de África ha sido cede de un intercambio constante entre poblaciones locales, migrantes del interior del continente y extranjeros, pues ha sido un centro de comercio establecido al menos desde 
el siglo VIII. ${ }^{2}$ Este intercambio llevó a la formación de la población y la cultura swahili con influencias tanto locales cuanto externas.

Parte de las influencias externas más importantes ha sido la introducción del Islam después del siglo VIII, lo cual muchas veces se ha equiparado con un cambio en las relaciones de género. Pero Askew (1999: 92) argumenta que las relaciones de género se vieron sobre todo afectadas en siglos más recientes con cambios no sólo por influencia del Islam, sino también por cambios en el sistema educativo, político y económico. Estos cambios han llevado a que la fuerza económica y política que tenían las mujeres se ha ido perdiendo en un sistema de herencia de tierras originalmente matrilineal, o donde al menos tenían una presencia importante por las prácticas matrimoniales uxorilocales (Askew, 1999: 87). Este cambio en las relaciones de género necesariamente causa tensiones que se ven reflejadas en los cuentos y los mitos de los swahili.

En el cuento se ve una estructura de herencia patrilineal: la hermana no pide más que bendiciones de sus padres, y el hermano en cambio se queda con todos los bienes materiales. El hermano además es egoísta con su hermana, argumentando que ella ha elegido su camino. Esta acción justifica la herencia patrilineal de los bienes materiales: las mujeres de alguna manera han escogido su camino al seguir su papel de hermanas, hijas y esposas devotas, y por ello el hermano responde: "I shall not [leave her anything]. I asked for the property, she asked for a blessing" (Steere, 1999: 302). Pero esto también es criticado al demostrar el extremo innecesario y egoísta al que llega el hermano para quitarle todos los bienes a su hermana. En cuanto se percata de que ella está obteniendo alimento a cambio de prestar los pocos utensilios de cocina que él le dejó, se los roba y la deja con la casa vacía, salvo por una sola semilla de calabaza. Los objetos en sí son también simbólicos ya que los utensilios de cocina son objetos femeninos, así como la semilla de calabaza, que representa la agricultura y la tierra trabajada por las mujeres y la cual anteriormente representaba su inde-

2 Hay varias polémicas alrededor de la concepción de los swahili: si deben de determinarse como un grupo étnico o no, y de sus orígenes. Para mayor información acerca de su historia y origen véase Allen (1982); Arens (1975); Berg (1968); Eastman (1971 y 1988); Nurse y Spear (1985); y Pouwels (2001 y 2002). 
pendencia del hombre. Pero el hermano le quita ambos, lo que refleja el exceso del sistema patriarcal que se ha impuesto sobre las mujeres y que las ha limitado no sólo en tener sus propios bienes, sino también en su manera de obtenerlos.

El evento más violento es cuando el hermano le destroza la planta de calabazas que había crecido mágicamente y que mantenía a la joven. En esta ocasión la que incita el acto es la esposa del hermano, que destruye la planta y despoja a su hermana de todos sus bienes y la mutila. Esta acción simboliza la violencia con la que las mujeres fueron despojadas de sus bienes y de su situación política y social. Ya no es parte de su hogar, ya que bajo los lineamientos patriarcales ella al tener edad de casarse debe de salir de su casa e ir a la de su esposo, pero al no tener aún un esposo está incompleta.

Enseguida la joven sale de su aldea y después de cruzar el bosque encuentra otra aldea donde se refugia arriba de un árbol. El hijo del rey se reposa debajo del árbol y ella lo moja con sus lágrimas. Es en este momento que se le identifica como una joven extraordinariamente bella y el hijo del rey decide llevársela a su casa, pero escondida, a exigencia de la joven. El joven príncipe se enamora de ella y se casan. Poco después ella da a luz y el príncipe sale de viaje. Es en ese momento que el hermano de la joven escucha hablar de la princesa sin una mano y decide ir en busca de ella. Para este momento del cuento el hermano ha perdido todos sus bienes y decide robarle a su hermana no sólo sus bienes, sino el lugar que había adquirido entre la familia real. El hermano convence a los reyes de destituirla al argumentar que es una bruja: "Your child has married a woman who has lost a hand. She was run out of town because she was a witch! She kills every man she marries!" (Steere, 1999: 305). Con esto se ilustra también lo importante que es conocer la familia de la esposa o el esposo de la pareja. Un matrimonio no es cuestión de dos individuos, sino que es una alianza entre familias, por ello se enfatiza la necesidad de un matrimonio "bien fundado" y también demuestra la disparidad de los géneros en su postura en la sociedad. Aunque el hermano está mintiendo se le da más peso a su voz que a la de su hermana.

$\mathrm{Al}$ salir del castillo la joven encuentra una serpiente y aunque cree que la va a atacar, sucede lo contrario, la serpiente busca que la joven la proteja. En agradecimiento a la bondad de la princesa, la serpiente la lleva a su casa y en el 
camino la lleva a un lago donde le instruye que bañe a su niño. "She carried the boy, and went to wash him. The boy fell into the lake, she lost him in the lake [...] The snake said, "Put in both of your hands." The child of Adam put her hands in, and found her son. She held him. And she drew out her hand: it was sound again" (Steere, 1999: 306). La simbología fálica de la serpiente representa el ideal de la fuerza patriarcal: protege a la joven y después le brinda bienes materiales que la llevan a ser ahora un beneficio para la familia de su esposo. La simbología de la serpiente va más allá del símbolo fálico, pues también es un símbolo importante de fuerza y de vida, así como de poder mágico. La joven es el enlace entre el poder mágico que le confiere la serpiente y su esposo, a quien regresa "entera" gracias a su bondad, a su astucia y a su respeto por la serpiente. Su riqueza no lo obtiene por medio de la violencia ni el robo, como en el caso de su hermano, sino por medio de su obediencia y astucia, así como por su contacto con la magia.

Otro motivo particular es el agua, motivo que comúnmente se equipara con la feminidad. Chevalier determina que: "the symbolic significance of water can be reduced to three dominant themes: the source of life, means of purification, center of regeneracy" (cit. en Ayodo, 1994: 124). Al entrar en el agua simboliza tanto su renacimiento como el de su hijo. Ella simbólicamente vuelve a dar a luz a su hijo y de la misma manera se vuelve una mujer al recuperar su mano. En el momento de casarse con el príncipe no logró su posición como mujer, su hermano de cierta manera aún tenía influencia sobre ella, y no tuvo la fuerza para defenderse. Al proteger a la serpiente y probar su valor, se le integra nuevamente al sistema patriarcal que la había desterrado y también logra convertirse en mujer, obtiene la fuerza para regresar y contar su historia. Es interesante recalcar que aunque es por medio de la intermediación mágica de la serpiente, en realidad es el agua la que le restituye su mano a la joven y la que la purifica a ella y a su hijo. Es la fuerza femenina de la vida y la pureza la que la hace una mujer completa.

Por lo tanto en el cuento se muestra la vulnerabilidad de la mujer en una sociedad donde se le pone tanta importancia a la figura paterna y del esposo, al perder ambas figuras la joven queda destituida, es vulnerable y por ello es violentada por su hermano. Pero a través del cuento se ve también una crítica 
a este mismo sistema. El hermano representa la manera injusta y violenta en la que a las mujeres se les ha ido despojando de los derechos y la posición que tenían anteriormente. El hermano es en realidad la figura más débil del cuento, mientras que ella surge como la figura más fuerte y así se muestra la centralidad de la mujer en el seno familiar y también la astucia de las mujeres en responder a estas injusticias y a sus problemáticas. Por ello aunque se ve la importancia de seguir las estructuras sociales para que una mujer esté completa (es decir cumplir sus deberes, ser obediente y, de manera más importante, casarse y tener hijos), también muestra una crítica sutil de estas mismas estructuras sociales.

"Ali, the Sword and the Secret Charm" cuenta la historia de Ali, quien hereda una espada y medicina secreta de su padre. Su padre le instruye que si quiere acostarse con una mujer sin que ella sea su esposa, siempre debe de interponer la espada entre los dos. En la primera parte de su historia, Ali se queda a descansar en una casa donde una anciana lo invita a comer y descansar con su hija, descrita como una mujer particularmente bella. En estas primeras figuras femeninas se representa la maldad al ser la anciana una bruja poseedora de magia y su hija una serpiente disfrazada. La hija representa la tentación, pues además de ser bella, sugiere que Ali comparta su lecho. Al convertirse en una serpiente que busca matar a Ali, la hija personifica las consecuencias negativas de la seducción. La serpiente representa la maldad en la tradición islámica y también representa el pecado de la seducción femenina. Ali muestra la fortaleza masculina al escapar de estas mujeres asesinando a la serpiente con la espada que había interpuesto entre los dos. Por lo tanto, la mujer es castigada por su control sobre el poder mágico, y por quererse contraponer al hombre. Refleja también la equiparación que se hace entre la mujer y la magia tradicional, y entre el hombre y el Islam en varias concepciones de la sociedad swahili. En este caso también es un mensaje religioso en el que el Islam vence a las prácticas preislámicas de magia. No solamente mata a la serpiente, sino que Ali juzga a la bruja y la condena en frente de toda la corte por uso de magia. Así castiga a las mujeres de manera privada y pública, con lo que personifica y justifica el triunfo del sistema patriarcal, por una parte, y, por otra, la del Islam.

Las otras tres figuras femeninas que aparecen en el cuento son la reina, la hermana del rey y la princesa. Ali sorprende a la reina buscando tener un amo- 
río con el orfebre del reino, para ello mata a quien cree que es su esposo pero en realidad es su hermano. Después Ali salva a la princesa, quien le pide que comparta su lecho. Él de nuevo pone la espada entre los dos, lo cual observa la hermana del rey cuando va a ver a su sobrina en la mañana. De esta manera vemos tres representaciones de la mujer: la reina personifica la incapacidad de la mujer de controlar sus impulsos sexuales, su avaricia al ir en busca del orfebre y su capacidad de traición; la hermana del rey representa el papel que juega la mujer en la familia, sobre todo tomando en cuenta que es la hermana del papá de una niña la que generalmente está encargada de su educación sexual y su rito de paso (Topan, 1995; Caplan, 1976); y finalmente en la joven se refleja el papel de la debilidad femenina, así como la función de los matrimonios en crear alianzas al casarla con Ali en agradecimiento por protegerlos a ella de los ladrones y a él de su esposa.

Por lo tanto este cuento se enfoca más bien en fortalecer la imagen del hombre como el que pone orden en la sociedad, y muestra el peligro de que una mujer tenga el poder de la magia y el político. Justifica un sistema patriarcal y que el papel correcto de la mujer es el de cumplir sus papeles sociales, sus funciones familiares y como alianza entre diferentes familias y comunidades. Se muestra la importancia del honor y la honestidad de Ali en contraposición a la maldad femenina encarnada por la bruja, su hija y la reina, todas ellas representadas sobre todo por la seducción. Pero aun dentro de esta justificación patriarcal se muestra también la centralidad de la mujer en su familia y para los hombres. Es por medio de la intervención de la hermana del rey que a Ali se le libra de cualquier acusación de violentar el honor de la joven princesa y es a través de la princesa que Ali obtiene un reino.

El último cuento que se comentará es "The Island of the Snake and the Land of Gold". Comienza también con la muerte del padre de la joven heroína, le explica que ella no es realmente su hija, sino que tomó cargo de ella, y que ambos vienen de una tierra de oro. Ella permanece en la casa después de enterrar a su benefactor y al pasar el sultán por su hogar se enamora de ella y decide casarse con ella. Una noche la reina resuelve ir en busca de su tierra de origen, lo cual logra con la ayuda de su esposo. Al llegar a una isla entra al bosque sola, donde se encuentra con una serpiente grande: 
Suddenly she saw a long snake that raised its head, saying in her language: 'What do you want?' The girl told her story, and the snake said: 'I will help you, on condition that you give me your first baby and a hundred pots of honey.' The young queen agreed, and the snake told her how to reach the Land of Gold. It also promised that it would cause the king of that country to believe that she was his lost daughter (Knappert, 1970: 105).

Aquí se ve de nuevo la imagen de una joven reina que es beneficiada por una serpiente que le habla y la ayuda a obtener su lugar en la sociedad, así como una gran riqueza. Es vista como una figura benigna e intermediaria entre la magia (una serpiente con poderes sobrenaturales) y su comunidad. Pero además de esta relación con la magia, de nuevo se apunta hacia la conexión entre la mujer y la tierra. Ella va en busca de su tierra no sólo por curiosidad, sino para reclamar algo que es suyo.

La obtención de las riquezas de La Tierra del Oro no es sólo como premio por su obediencia, sino que ella misma encierra una cierta astucia. Aunque está rodeada de figuras de poder masculino, ella al final logra lo que buscaba y su hijo, gracias a ella, heredará tres reinos. Las mujeres jugaban un papel central al unir su herencia de tierras con la herencia de riqueza material y el estatus político que poseía el hombre (Nurse y Spear, 1985: 23). Por ello este cuento enfatiza las estructuras sociales, pero también conlleva un mensaje sutil de la fuerza femenina: la fuerza de la reina de enfrentarse a la serpiente y seguir sus órdenes, así como obtener, por medio de su contacto con la magia y la posición social de su esposo, riqueza y felicidad para ella y su familia.

Finalmente, en el caso de "La Épica de Liongo", aunque no parecen a primera instancia jugar un papel central en el cuento, las dos figuras femeninas centrales, la madre de Liongo y su esclava, ayudan a que Liongo logre sus hazañas. Liongo personifica una violencia mítica y un poder excesivo, por lo que lo hacen prisionero. Estando ahí le pide ayuda a su madre; por medio de su esclava, manda decirle que cocine unos pasteles y que en ellos esconda navajas para poderse escapar; la esclava le lleva el mensaje a su madre y ella le manda las navajas a su hijo. Es también su madre la que tiene que acercarse a su hijo al final de la épica para confirmar si está muerto o no y comunicarlo a su comunidad. 
Así las mujeres juegan un papel de intermediarias entre el poder, en este caso Liongo, y su comunidad. Ellas son las únicas que tienen la fuerza de acercarse a este poder excesivo y son las únicas que tienen la capacidad de mediar con él. Las figuras en sí son significativas, apuntan hacia la conexión particular entre madre e hijo, así como la relación íntima que se llegaba a tener con las figuras de los esclavos en la sociedad. Por medio de sus relaciones cercanas con Liongo estas mujeres son capaces de fungir como intermediaras entre él y su comunidad y también de ayudar al héroe.

Es así que además de este papel de intermediarias, juegan también un papel heroico. Mbele (2006: 62) argumenta que muchas veces en las épicas se ignoran las hazañas heroicas de las mujeres y se reconoce solamente su papel como intermediarias o su ingenio al enfrentarse a las crisis dentro de las épicas. En el caso de este mito se puede ver la actitud heroica de ambas mujeres. La esclava arriesga su vida al llevarle las navajas. Por otro lado, su madre es la que debe verificar que él está muerto, ella es la que tiene la fuerza para acercarse a él y debe de enfrentarse con la muerte de su único hijo. En ambas instancias, estas mujeres, que no parecen tener mucha fuerza ni posición en la sociedad, en realidad llevan a cabo hazañas heroicas en apoyo a Liongo y también en su papel como intermediarias. También estos personajes muestran una crítica a los excesos de Liongo, al mostrar la manera en que sus acciones no sólo ponen en peligro a la joven esclava, sino que también hacen sufrir a su madre al forzarla a enfrentar directamente lo que es una pérdida central en su vida, la pérdida de su hijo, de su única familia.

Así, en estos cuentos y esta épica swahili pueden verse reflejadas diferentes enseñanzas sociales, justificaciones de ciertas prácticas sociales y críticas de éstas. En las narraciones se muestran figuras femeninas fuertes y otras más débiles, todas ellas con un papel particular. Es problemático llegar a una conclusión general acerca del papel de la mujer dentro de los cuentos, pues los mensajes sociales y filosóficos varían, lo que muestra que, como argumenta Hountondji, no hay una única filosofía para una población, sino que hay varias voces femeninas, y con ellas se ven tanto transmisiones de las tradiciones orales como su subversión. 


\section{Conclusión}

En este trabajo se muestra una revisión de la función filosófica, social y educativa de la tradición oral en sus expresiones africanas con la finalidad de presentarla como parte de la literatura. La finalidad de esta discusión es para llegar a un análisis literario de los cuentos y la épica swahili. Con esto se buscó demostrar que no hay un solo discurso que atraviesa toda la tradición oral swahili y aún menos todas las tradiciones africanas. La oralidad funge un papel particular en las culturas africanas al integrar elementos filosóficos, sociales, educativos y artísticos. Claramente debe de incluirse dentro de la concepción de literatura ya que conlleva una representación estética, un mensaje social y artístico, y juega una función filosófica en las poblaciones donde es representada. Parte de lo que lo hace literatura es la representación por narradores y narradoras que constantemente recuentan las historias, con lo que demuestras que es una tradición múltiple y cambiante.

Las mujeres juegan un papel central en este aspecto ya que ellas son muchas veces las narradoras de estas expresiones orales, en contextos informales y formales, privados y públicos, pero a través de todos ellos entran en diálogo con la oralidad y con su audiencia. Es por medio de sus narraciones que las mujeres demuestran su función no sólo de depositarias de esta tradición, sino también como compositoras y como subversoras. Son compositoras en el sentido de que cada representación de un cuento es particular, pero también en el sentido en que ellas mismas "escriben" sus poemas, sus cantos o sus cuentos, e incluyen en ellos muchas veces un cuestionamiento de las enseñanzas sociales y filosóficas que justifican la represión femenina. Por medio de estas voces femeninas se incluyen visiones alternativas y subversiones de las tradiciones o de prácticas más recientes que están amenazando su posición social.

Dentro de los cuentos las mujeres juegan diferentes papeles; los personajes femeninos generalmente justifican ciertas prácticas sociales, muestran la imagen de la mujer ideal o de su contrario, de la maldad femenina. Por medio de los cuentos y los mitos se muestran las diferentes voces femeninas que forman parte de la sociedad en cuestión, mostrando que no hay una sola visión de la mujer africana; asimismo se presenta la debilidad femenina, su fortaleza, su relación 
con el poder mágico y político, su papel central en la familia, su astucia y en ocasiones también su falta de inteligencia. Las concepciones acerca de la mujer en estas sociedades se desglosan, pero también en ocasiones se incluyen críticas fuertes a estas concepciones. Como se mostró con el caso específico de los cuentos swahili, aunque las mujeres no sean los personajes principales de los cuentos o las épicas, sus acciones o su posición en la sociedad resultan centrales para el héroe o la heroína. Ellas también llevan a un cuestionamiento, y en ocasiones a una subversión de los papeles sociales que se les asigna y de la filosofía detrás de dichas estructuras patriarcales. Por lo tanto, en respuesta a la pregunta central de este artículo se puede concluir que las funciones de la mujer en tradiciones orales africanas son múltiples, pero principalmente tienen una función como transmisora y como figura subversiva.

\section{Referencias bibliográficas}

ABBAS H. EL-NOUR, Eiman (2011): "Not just a pretty face: Women as storytellers and subjects in the folktales of Northern Sudan". En Tydskrif Vir Letterkunde. Núm. 48 (2), pp. 171-185.

ASKEW, Kelly M. (1999): "Female Circles and Male Lines: Gender Dynamics along the Swahili Coast”. En Africa Today. Núm. 46 (3/4), pp. 67-102.

AYODO, Awour (1994): “Definitions of the Self in Luo Women's Orature”. En Research in African Literatures. Núm. 25 (3). pp. 121-129.

CAPLAN, A. P. (1976): “Boys' Circumcision and Girls' Puberty Rites among the Swahili of Mafia Island Tanzania". En Africa: Journal of the International African Institute. Núm. 46 (1), pp. 21-33.

FINNEGAN, R. (1998): Tales of the City: A Study of Narrative and Urban Life. Cambridge: Cambridge University Press.

FINNEGAN, R. (2007): The Oral and Beyond: Doing Things with Words in Africa. Oxford: James Currey. 
GUEYE, Marame (2010): “Woyyi Céet: Senegalese Women’s Oral Discourses on Marriage and Womanhood". En Research in African Literatures. Núm. 41 (4), pp. 65-86.

HOUNTONDJI, Paulin J. (1977): Sur la "Philosophie Africaine" critique de l'ethnophilosophie. París: François Maspero.

HOUNTONDJI, Paulin J. (2004): “Knowledge as a Development Issue”. En K. Wiredu (ed.), A Companion to African Philosophy. Malden: Blackwell, pp. 529-537.

JULIEN, Eileen (1971): African Novels and the Question of Orality. Bloomington: Indiana University Press.

KNAPPERT, Jan (1970): Myths and Legends of the Swahili. Londres: Heinemann Educational Books.

LORENTZON, Leif (2007): “Is African Oral Literature Literature?” En Research in African Literature. Núm. 38 (3), pp. 1-12.

MAFEYE, Archie (1997): "The Anthropology and Ethnophilosophy of African Literature”. En Alif: Journal of Comparative Poetics. Núm. 17, pp. 6-21.

MBELE, Joseph L. (1996): “Wimbo wa Miti: An Example of Swahili Women's Poetry". En African Languages and Cultures. Núm. 9 (1), pp. 71-82.

MBELE, Joseph L. (2006): “Women in the African Epic". En Research in African Literature. Núm. 37 (2), pp. 61-67.

MIRZA, Sarah y Margaret Strobel (eds.) (1989): Three Swahili Women: Life Histories from Mombasa, Kenya. Indiana: Indiana University Press.

MUSIYIWA, Mickias (2009): "Displacement of African Women from Storytelling: Implications for the Development of Modern African Children's Literature". En Sankofa. Núm. 8, pp. 6-15.

NGWAINMBI, Emmaneul K. (2007): “Power of the Spoken Word: Journeying Back to Collect Kom Folktales". En Sankofa. Núm. 6, pp. 20-29. 
NNAEMEKA, Obioma (1994): "From Orality to Writing: African Women Writers and the (Re)Inscription of Womanhood". En Research in African Literatures. Núm. 25 (4), pp. 137-157.

NURSE, Derek y Thomas Spear (1985): The Swahili: Reconstructing the History and Language of an African Society 800-1500. Pennsylvania: University of Pennsylvania Press.

RAJENDRA PRASAD, Ch. A. (2007): "Logocentrism and a priori Binary Opposition vis-à-vis Women”. En Fabula. Núm. 48 (1/2), pp. 100-109.

ROSENBERG, Aaron L. (2012): "Remembered Intimacies: Tradition and Gendered Power in Tanzanian Creative Expression”. En Research in African Literature. Núm. 43 (1), pp. 118-135.

SCHEUB, Harold (1999): The African Storyteller: Stories from African Oral Traditions. Iowa: Kendall/Hunt Publishing Company.

SOUGOU, Omar (2008): “Transformational Narratives: Hearing/Reading Selected Senegalese Folktales by Young Women". En Research in African Literature. Núm. 39 (3), pp. 26-38.

STEERE, Edward (1999): “The Girl with One Hand”. En Harold Scheub (ed.), The African Storyteller: Stories from African Oral Traditions. Iowa: Kendall/Hunt Publishing Company.

SULLIVAN, Joanna (2006): "Redefining the Novel in Africa". En Research in African Literature. Núm. 37 (4), pp. 177-188.

WA THIONG'O, Ngugi (2007): "Notes towards a Performance Theory of Orature". En Performance Research. Núm. 12 (3), pp. 4-7.

TOPAN, Farouk (1995): "Vugo: A Virginity Celebration Ceremony among the Swahili of Mombasa”. En African Languages and Cultures. Núm. 8 (1), pp. 87-107.

VIVEROS, Akuavi Adonon y Fabien Adonon Djogbénou (2009): Escrito en las nubes: Inmanencia de la tradición oral en África negra. México: Universidad Nacional Autónoma de México. 
WEST, M. Genevieve (2007): “'Creating a Change in Continuity”: A Conversation with Makuchi, Author of The Sacred Door and Other Stories: Cameroon Folktales of the Beba". En Obsidian III: Literature in the African Diaspora. Núm. 8 (2), pp. 53-66.

WIREDU, Kwasi (2009): "An Oral Philosophy of Personhood: Comments of Philosophy and Orality". En Research in African Literature. Núm. 40 (1), pp. 8-18. 